\title{
In-situ rock mass stress-state measurements in scales of mineral deposits: problem-solving
}

\author{
Alexander Balek ${ }^{1^{*}}$ and Anatoly Sashourin ${ }^{1}$ \\ ${ }^{1}$ Mining Institute of Urals Division of Russian Academy of Sciences, Yekaterinburg, \\ Russia.
}

\begin{abstract}
This paper describes the new method for in-situ measurements of the most large-scale stress fields that are comparable to dimensions of deposits and mining leases. The method considers open cast mines and collapse zones of underground mining as disturbing cavities. The calculation of stresses is carried out thru measured deformations for the conditions of plane stressed state from the measured displacements of survey marks on the earth surface. GPS or GLONASS technology is used for the measuring. The results of in-situ study deformations of pit edges Pipe Udachnaya Mine (Sakha Republic, Russia) have been presented as an example of practical application of the method.
\end{abstract}

\section{Justification of in-situ stress state measurements technique}

The most of modern deformative methods of the stressed state rock mass in-situ measurements are based on calculation of the stresses according to Hooke's law from the measured deformations caused by purposeful stress field distortions. Ordinarily for this purpose we use the redistributions of stresses resulted of the excavated from the rock mass stressed cavities with known shape and size such as boreholes, crevices, mine workings, worked-out areas, etc. It is assumed that in calculations of the stresses there can take place elastic deformation of continuous homogeneous isotropic medium in a uniform stress field [1-3].

Mining Institute of Urals Division of Russian Academy of Sciences for in-situ measurements of the most large-scale (among geomechanics objects) stress fields that are comparable to the capacity of deposits and mining leases considers open cast mines and collapse zones of underground mining as disturbing cavities [4]. The elastic deformations of the surrounding rock mass are determined by the measurements of displacement of the survey marks which were pre-installed in the field of the future of elastic deformation in the affected zone of the taken out cavity, but out of the trough of rock mass gravitational settling [5-6].

As far as in-situ measurements are aimed at identifying the tectonic stresses in the horizontal plane, the calculation of stresses is carried out thru measured deformations for the conditions of plane stressed state from the measured displacements of survey marks on the earth surface. GPS or GLONASS technology is used for the measuring [7-12]. Open

\footnotetext{
*Corresponding author: balek@igduran.ru
} 
cast mines and collapse zones are approximated by elliptical holes which are taken out in a stressed thin plate.

The displacements, according to the superposition principle, caused by the excavation in elastic isotropic plane of elliptical hole, can be represented as the difference between the displacements, caused by loading of plane with a hole, and the displacements caused by loading of the plane without hole.

Numerical values of displacement vectors for survey marks on the earth surface are determined by analytical dependences which are developed on the basis of the complex variables. The solution of combined equations, that are composed on combinations of measured displacement vectors of survey marks, allows to evaluate unknown parameters of the stressed state.

The difficulty lies in the fact that not all of the required unknowns are independent physical parameters. Having data about the geometry of the disturbing cavity and about displacements of the surrounding massif with an arbitrarily large number of measured vectors and with any of their locations it is impossible to determine:

- the values of the principal stresses $\sigma_{1}$ and $\sigma_{2}$ without knowing Young's modulus of the massif (or vice versa);

- the ratio of the principal stresses $\sigma_{1}$ and $\sigma_{2}$ without knowing the value of the Poisson's ratio of the massif (or vice versa).

The proposed technique is aimed to resolve this problem.

Analysis of the equations, which are linking changes in the geometry of the disturbing cavity with the displacements of surrounding massif, shows that the influence of the Poisson's ratio $\mu$ at solving equations does not exceed few percent. If the changes characterize the rocks this influence is insignificant and the Poisson's ratio is in the range $\mu$ $=0.2 \div 0.3$. Adopting Poisson's ratio as the predetermined value we can find the remaining unknown parameters by the follow two methods.

Method 1. By setting different Young's modulus $E$ for each vector of measured horizontal displacements the solution of the two equations system determine the ratio of principal normal stresses $\sigma_{2} / \sigma_{1}\left(\sigma_{2}\right.$ corresponds to the maximum compression) and their orientation by the angle $\alpha$ (that is axes azimuth $\sigma_{2}$ ).

A comparative analysis of the received data variation for different values of the Young's modulus $E$ is performed after finding the initial massif stresses of all measured vectors. As a result, the value of the modulus and ratios of the principal normal massif stresses $\sigma_{2} / \sigma_{1}$ and their orientation are taken so (appropriate to this modulus) that the difference between the standard deviation of the calculated angles $\alpha$ and their arithmetic means is minimized.

The method is very remarkable for comparative simplicity and the ability to find all the unknown parameters through rigorous analytical solutions. However, it has the drawback due to the nonlinearity of basic relationships. Regardless of the reference point position and a vector of its displacements with increasing of default values of the Young's modulus the calculated direction of major axes of the stress tensor $\sigma_{2}$ quickly approach to the azimuthal direction of the point. The final result appears to be dependent on the uniformity of the metering points distribution around the cavity.

And with this method Young's modulus of the surrounding massif is taken the same for all metering points. This assumption requires justification too.

Method 2. By selection of pairwise combinations of measured vectors (i.e. solution of system of the four equations) the three unknown parameters are determined: the ratio of the principal stress $\sigma_{2} / \sigma_{1}$, the direction of their axes through the angle $\alpha$, and the massif Young's modulus $E$ (defined as a fraction of maximum stresses: $E / \sigma_{2}$ ).

The calculations are carried out in two stages in order to facilitate the task and make the analysis of the results more convenient. Initially is defined the set of possible values of $E$ 
and $\sigma_{2} / \sigma_{1}$ over a range of axes azimuths $\sigma_{2}$ from $0^{\circ}$ to $180^{\circ}$ by the solution of two equations system for each of the measured vectors. These equations satisfy the criterion of compressive stresses existence subject to the condition of maximum value $\sigma_{2}$.

Further calculations are reduced to finding such pairwise combinations of displacement vectors, which have the same direction of the principal stress axes at the same values of $\sigma_{2} /$ $\sigma_{1}$ and $E$. It is obviously that such task as this has no analytic solution. It can only be solved numerically because the task represents finding of the three curves crosspoint in threedimensional space or finding of four independent nonlinear functions $f\left(\alpha, \sigma_{2} / \sigma_{1}\right)$ and $f(\alpha$, $E$ ) for each pair of vectors in two dimensional space.

In practice this solution is more convenient to carry out graphically. It is possible to draw the curves on the computer screen and to identify common angles $\alpha$ with a given precision for the crosspoints of these functions on two-dimensional graph by 3 axes: $\alpha-\left(\sigma_{2}\right.$ $/ \sigma_{1}$ ) and $\alpha-E$. In this case angle $\alpha$ is set to a specific level of precision (for example: $\pm 5^{\circ}$ ) and with really possible range of relationships between the principal stresses, for example: $1 \leq\left(\sigma_{2} / \sigma_{1}\right)<5$.

\section{Examples of practical application of technique}

According to the in-situ measurements analysis of "Udachnaya" pit edges deformations Mining Institute of Urals Division of Russian Academy of Sciences was first who obtained experimental data about the initial stresses field and the relative characteristics of the massif deformation around deposit "Udachnaya pipe" on the bases of several kilometers. For this purpose there were used the results of instrumental GPS monitoring carried out by LLC "GENESIS CENTER" for the period 1997 to 2009. There were analyzed vectors of horizontal displacements of those bench marks that reflect the deformation of massif from pit excavation and those bench marks which are not affected by local deformation symptoms caused by "not geomechanical" factors: subsidence of edges and separated rock blocks, the processes of freezing, thawing, swamping etc. According to the analyzed results the legitimacy of analytical methods and of models of continuous elastic medium mechanics was substantiated for the displacement vectors of 29 bench marks which are shown in Figure 1.

On the 3D model of the earth's surface there is the outer boundary of "Udachnaya" pit with the size 2000 to 1900 meters and the boundary of its bottom with the size 600 to 260 meters. The excavated from 1997 to 2009 rock volumes (about 40 million cubic meters) were approximated by a cavity in form of cylinder with the vertical depth $h=160$ meters and with ellipse base. The ellipse semi-axes are: $a=300$ meters; $b=130$ meters (Fig. 1). The coordinate $X$-axis is directed from west to east, and coincides with a major semi-axis $a$. The $Y$-axis is directed from south to north and coincides with the minor axis $b$.

The bearing strata is represented by sandy, clay and algal limestones and dolomites, which are weak and rather weak, with ultimate strength on uniaxial compression of 10 to 35 $\mathrm{MPa}$ and 4 to $10 \mathrm{MPa}$, respectively. According to degree of disturbances the rock massif is divided as follows:

- $\quad$ by eastern edge - the disturbance is very high (more than 12 joint systems);

- $\quad$ by south edge - the disturbance is high (10 - 12 joint systems);

- $\quad$ by south-west and west edges - the disturbance is medium ( 8 - 10 joint systems );

- $\quad$ by the northern edge - low disturbance with $6-8$ joint systems. 


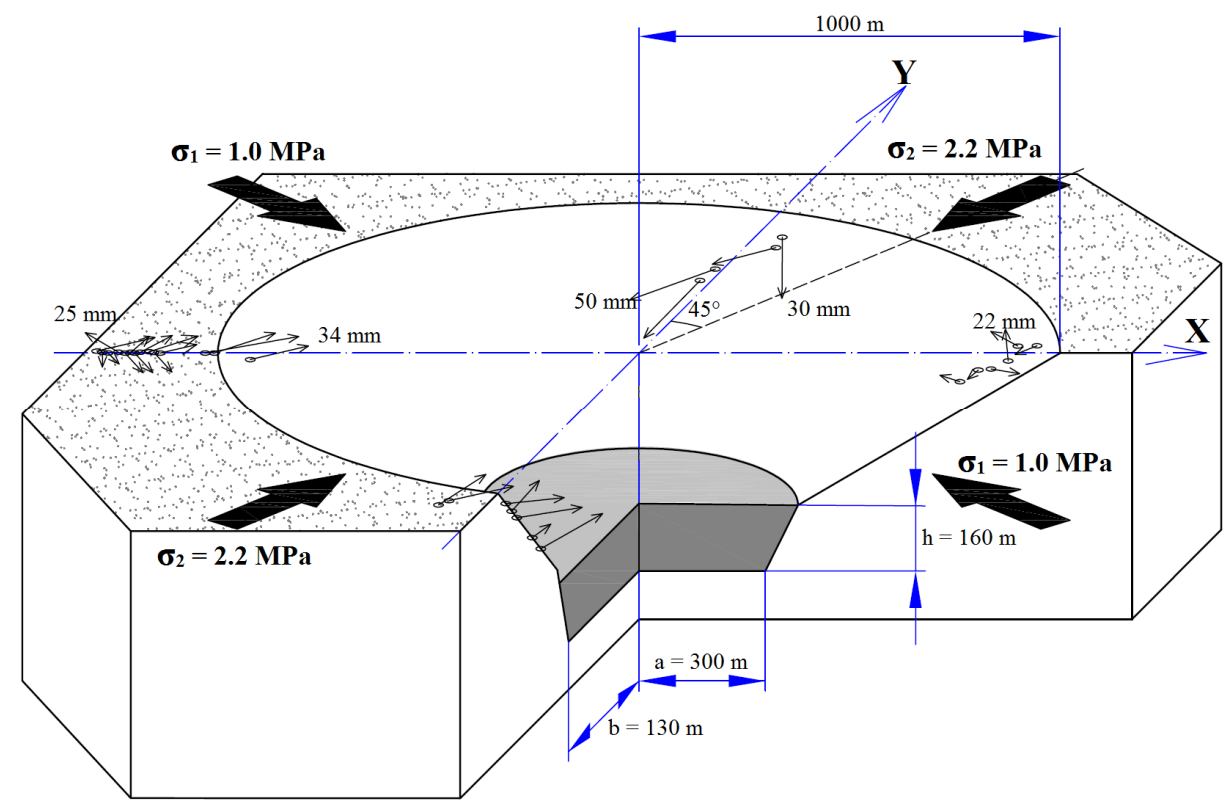

Fig. 1. Scheme for in-situ measurements and for calculation of the initial stresses of massif around the excavated volumes of pit "Udachnaya" in the period from 1997 to 2009. These volumes were approximated by an elliptical cylindrical cavity.

The calculations performed with the both the above-stated methods for the following boundary conditions: $a=130$ meters, $b=300$ meters, $\mu=0.3, \sigma_{1}=-1 \mathrm{MPa}$.

Solution of equation systems by the first method (i.e. with equation roots $\sigma_{2} / \sigma_{1}$ and $\alpha$ by enumeration of possibilities of Young's modulus $E$ ) gave us 29 pairs of equation roots satisfying the above-stated criterion. Comparative analysis of the obtained angles $\alpha$ showed that the minimum standard deviation (that was $52^{\circ}$ ) is reached at $E=1700 \mathrm{MPa}$.

The results of solving the same equations system by the second method (finding the equation roots $E$ and $\sigma_{2} / \sigma_{1}$ by carrying out comparative analysis of the pairwise combinations of 29 bench marks ) showed that in the range $1 \leq\left(\sigma_{2} / \sigma_{1}\right)<5$ with an accuracy of $\pm 5^{\circ}$ common azimuths of axis $\sigma_{2}$ are available in 48 crosspoints of the considered functions $f\left(\alpha, \sigma_{2} / \sigma_{1}\right)$ and $f(\alpha, E)$.

Obtained by the first method summary diagram which represents the calculated ratios $\sigma_{2} / \sigma_{1}$ and orientation of the principal axes of the massif initial stresses is shown in Figure 2.

A similar diagram for the obtained by the second method results is shown in Figure 3 with the values of Young's modulus $E$, defined for the relevant sections of the massif. 

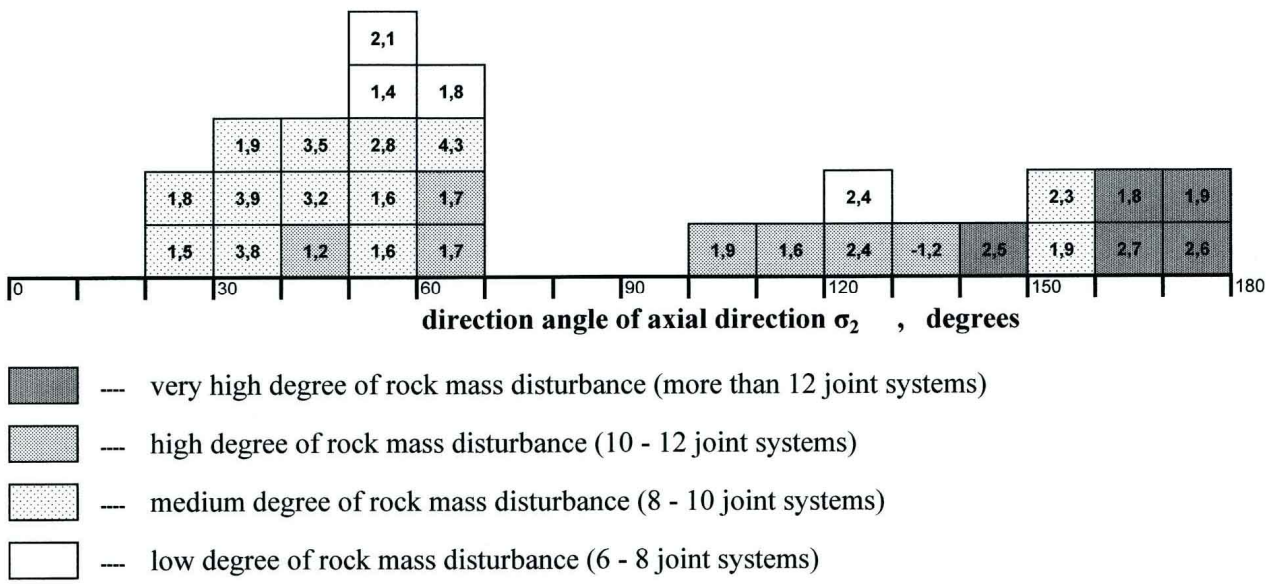

Fig. 2. Diagram with the calculation results of ratio $\sigma_{2} / \sigma_{1}$ and orientation of the principal axes of the tensor of rock massif stresses in the vicinity of the pit "Udachnaya" for each of the 29 bench marks at $\sigma_{1}=-1 \mathrm{MPa}$ and the massif Young's modulus $E=1700 \mathrm{MPa}$. On the horizontal axis in the azimuthal range $0^{\circ}-180^{\circ}$ with step $10^{\circ}$ there are azimuths of axial direction $\sigma_{2}$, and on a vertical axis there is identification frequency (about the relevant direction) with the calculated values of $\sigma_{2} / \sigma_{1}$ and degree of the massif disturbance in the area of one of the bench marks.

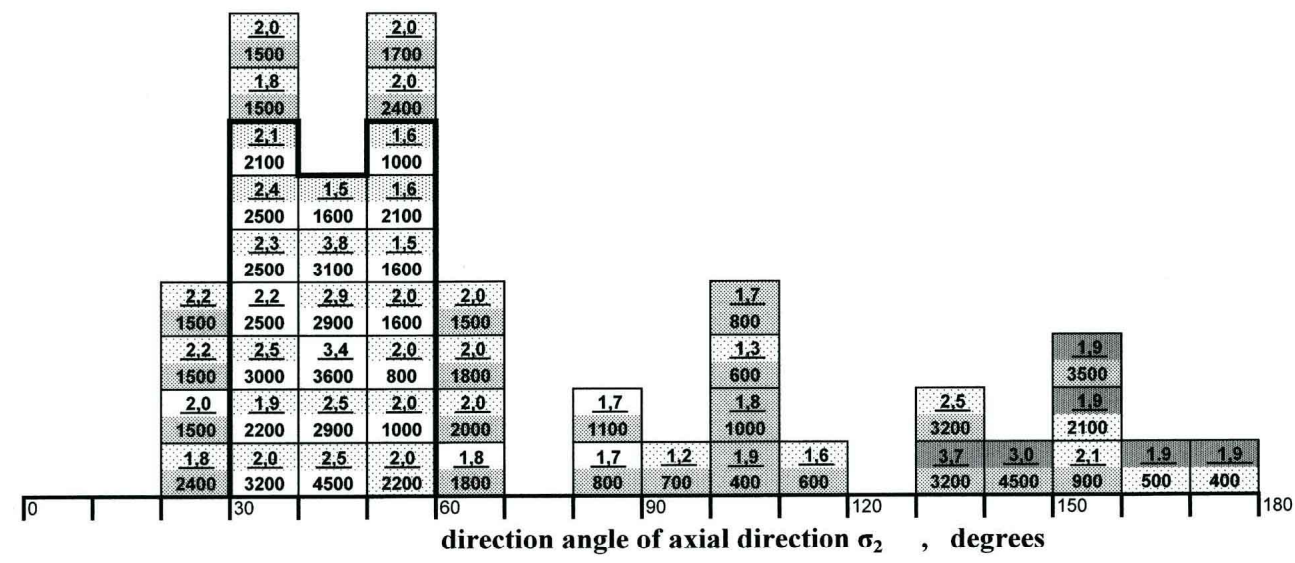

$$
\begin{aligned}
& \text {-- very high degree of rock mass disturbance (more than } 12 \text { joint systems) } \\
& - \text { - high degree of rock mass disturbance (10 - } 12 \text { joint systems }) \\
& - \text { - medium degree of rock mass disturbance ( } 8-10 \text { joint systems }) \\
& \square-\text { - low degree of rock mass disturbance }(6-8 \text { joint systems })
\end{aligned}
$$

Fig. 3. Diagram with the calculation results of ratio $\sigma_{2} / \sigma_{1}$, modulus $E$ and orientation of the principal axes of the initial stresses tensor of rock massif in the vicinity of the pit "Udachnaya" for each of the 48 pairwise combinations of the bench marks. On the horizontal axis in the azimuthal range $0^{\circ}-180^{\circ}$ with step $10^{\circ}$ there are azimuths of axial direction $\sigma_{2}$. On a vertical axis there is identification frequency (about the relevant direction) with the calculated values $\sigma_{2} / \sigma_{1}$ (represented in the numerator), modulus $E$ (represented in the denominator as fracture of $\sigma_{1}$ ) and degree of the massif disturbance in the area of one of the bench marks (represented by filling). 


\section{Discussing the results and conclusions}

In both methods were obtained qualitatively identical results. Prevailing ratio of principal stresses of the surrounding rock massif $\sigma_{2} / \sigma_{1}$ was close to 2.2 , and in the distribution of the calculated directions of the of maximum compression axis $\sigma_{2}$ appeared two statistical samples: prevailing sample at the north-east direction and less clearly defined at the southeast direction.

For the analysis of independence of the variables by criteria $\left\langle\chi^{2} »\right.$ there are $n=29$ of calculated differences between axis azimuth $\sigma_{2}$ and direction angle defined by elliptic coordinate of the appropriate bench mark. These differences were compared to the expected frequency of their uniform distribution with the arithmetic mean difference of $67^{\circ}$. The obtained $\chi^{2}=381$ for $(n-1)=28$ degrees of freedom even for a significance level of $0.05 \%$ is 6.5 times higher than the table value $\chi^{2}=59.3$. This indicated significant independence of identified directions of axes $\sigma_{2}$ by the uneven distribution bench marks around the pit.

Comparative analysis indicated statistical significance of both samples of calculated axis azimuths $\sigma_{2}$, because the mean square error of difference $S_{d}$ between sets of northeastern and south-eastern directions was: for the 1 st method $S_{d}=7.6$ and for the 2 nd method $S_{d}=7.8$. Received mean square errors are less than the differences between the mean values of azimuths (which are $80^{\circ}-95^{\circ}$ ) for more than an order of magnitude.

This does not allow to exclude samples of southeast direction from consideration and therefore to make a clear conclusion about prevailing direction of the greatest compression $\sigma_{2}$ in the initial stresses field of the surrounding rock massif. The problem was solved by using the calculated modules of elasticity of different parts of the massif determined by the 2nd method and geological data about their disturbance.

The diagram in Figure 3 shows that northeastern sample belongs to areas with medium and low disturbances. The highest disturbance concentrates in the extreme south-eastern sector. This gave grounds for advancing a working hypothesis that the southeastern sample (that is more statistically "weak" and with more variability) is causal inference of the deformation processes of fractured sections of the massif. Hierarchically block medium directed chaotic shifts of rock blocks and bench marks.

By the criterion of rock disturbance in the installation site of a benchmark the whole array of the 2 nd method from $n=48$ calculated data was divided into $m=2$ statistical samples:

1st sample: 20 pairs of bench marks installed in areas with extremely low and medium degree of disturbance (6 - 10 joint systems) with an arithmetic mean value of the modulus $E=2345 \sigma_{1}$;

- 2nd sample: 28 pairs of bench marks in areas that include high and very high disturbance (more than 10 joint systems) with an average modulus $E=1621 \sigma_{1}$.

The variance between samples $S_{B}^{2}$ with the degree of freedom $(m-1)=1$ was $S_{B}^{2}=$ $6290112 \sigma_{1}$, and variance within sample $S^{2}{ }_{W}$ with degrees of freedom $(n-m)=46$ is not exceeded $972,101 S^{2}{ }_{W}=\sigma_{1}$. The ratio between the variances $F=S_{B}^{2} / S_{W}^{2}=6,47$ is more than table value of Fisher criterion $F=4.05$ for the significance level of 5\%.

This allowed to accept the hypothesis of different sources that form variability the samples and therefore to consider only the results obtained by 20 pairs of bench marks in areas with low and medium disturbance for determining the parameters purpose of the initial stress field (in Figure 3 are shown in bold line). According to the given bench marks the calculated axis azimuths $\sigma_{2}$ are concentrated in a narrow sector from $31^{\circ}$ to $60^{\circ}$. Calculated by the remaining bench marks azimuths are uniformly distributed in the sector from $22^{\circ}$ to $171^{\circ}$ (Fig. 3).

Thus, as a result we obtained the following generic parameters. 
The measured parameters of the rock mass stressed state of deposit Udachnaya Pipe (for assumed 95\% confidence interval for the Poisson's ratio 0.3 ) are:

- the ratio between principal initial stresses of the rock mass is

- the azimuth of principal direction $\sigma_{2}$ is

$$
\sigma_{2} / \sigma_{1}=2.2 \pm 0.3 \text { (with } S=0.6 \text { ); }
$$

- Young's modulus of the rock mass is

$$
45^{\circ} \pm 4.4^{\circ} \quad\left(\text { with } S=10.1^{\circ}\right) \text {; }
$$

$$
E=2345 \sigma_{1} \pm 410 \sigma_{1} \quad\left(\text { with } S=931 \sigma_{1}\right)
$$

Where $S$ is the standard deviation of relevant parameter.

The offered technique was first that allowed to obtain experimental data about initial stress-strain state and the elastic characteristics of the rock massif at the most large bases (for space it is about $5-10 \mathrm{~km}$, for time it is about 10 years), and to consider statistical different degree of significance of in-situ measurements results caused by hierarchical block structure the deformed rock strata.

\section{References}

1 Kodama J., Miyamoto T., Kawasaki S., Fujii Y., Kaneko K. et al. International Journal of Rock Mechanics \& Mining Sciences. 63. P. 1-11. (2013).

2 Figueiredo B., Cornet F. H., Lamas L., Muralha J. International Journal of Rock

Mechanics \& Mining Sciences. 72. P. 37-48. (2014).

3 Wittke, W., Sykes R., Semprich S., Plischke B. Rock Mechanics, Theory and Applications with case histories, Springer-Verlag, Springer; 1 edition, 1076 p (1990).

4 A.Ye. Balek. Rock mechanics- a challenge for society: Proceedings of the ISRM regional symposium EUROCK 2001. P. 807-812. (2001).

5 Geertsma J. The Homogeneous Case. Verh. Con. Ned. Geol. Mijnbouwk. Gen. 28. p. 4362. (1973).

6 Sainoki A., Mitri H. S. International Journal of Rock Mechanics \& Mining Sciences. 66. P. 19-29. (2014).

7 C. Liu, J. X. Gao, X. X. Yu, J. X. Survey Review. 47. P. 79-86. (2015).

8 Yan Bao, Wen Guo, Guoquan Wang et al. Journal of Surveying Engineering. 144. (2017).

9 Zanutta, A., Negusini, M., Vittuari, L. et al. Journal of Geodynamics. 110. P. 31-42. (2017).

10 Ludovico Biagi, Florin Calin Grec, Sensors. 16. P. 21-40. (2016).

11 Yigit C.O., Coskun M.Z. Yavasoglu H. et al. Measurement. 91. P. 398-404. (2016).

12 Mohtarami E., Jafari A., Amini M. International Journal of Rock Mechanics \& Mining Sciences. 67. P. 43-56. (2014). 